\title{
The strength of small-instanton amplitudes in (deconstructed) gauge theories with compact extra dimensions
}

\author{
Erich Poppitz \\ Department of Physics, University of Toronto \\ Toronto, Ontario, M5S1A7, Canada \\ E-mail: poppitz@physics.utoronto.ca

\section{Yuri Shirman} \\ Department of Physics, California Institute of Technology \\ Pasadena, CA 91125, USA \\ E-mail: yuri@theory.caltech.edu
}

ABSTRACT: We study instanton effects in theories with compact extra dimensions. We perform an instanton calculation in a $5 \mathrm{~d}$ theory on a circle of radius $R$, with gauge, scalar, and fermion fields in the bulk of the extra dimension. We show that, depending on the matter content, instantons of size $\rho \ll R$ can dominate the amplitude. Using deconstruction as an ultraviolet definition of the theory allows us to show, in a controlled approximation, that for a small number of bulk fermions, the amplitude for small size instantons exponentially grows as $e^{\mathcal{O}(1) R / \rho}$.

Keywords: Field Theories in Higher Dimensions, Nonperturbative Effects, Solitons Monopoles and Instantons, Extra Large Dimensions. 


\section{Contents}

1. Introduction, summary, and outlook 1

1.1 Introduction

1.2 Summary 2

1.3 Outlook

2. Instantons in compactified 5d gauge theory: the supersymmetric case 1

2.1 Exact results in compactified 5d supersymmetric theory and semiclassical calculations

2.2 The $\rho$ dependence of the instanton amplitude in compactified supersymmetric theories

3. A brief review of deconstruction

4. Instantons in compactified 5d gauge theory: the non-supersymmetric case

4.1 The "step-function" approximation to massive instanton determinants

4.2 Deconstructing the instanton amplitude in a compactified pure gauge theory

4.3 Bulk matter and the instanton amplitude

5. Corrections to the step-function approximation

\section{Introduction, summary, and outlook}

\subsection{Introduction}

Theories with extra dimensions may play a role in resolving many issues in particle physics, from the hierarchy problem [1] and supersymmetry breaking [2, to the flavor problem and proton stability [3]. In many models, the extra dimensions are accessible to Standard Model gauge fields. If gauge fields are allowed into the extra dimensions, nonperturbative effects seen in the low-energy $4 d$ theory may substantially differ from those in ordinary $4 \mathrm{~d}$ theories. In $4 \mathrm{~d}$ gauge theories, asymptotic freedom of the gauge coupling typically suppresses the contributions of small instantons to instanton amplitudes, ensuring that the axion potential and the amplitudes of $\Delta B=\Delta L=3$ processes in the Standard Model are independent of the ultraviolet physics [ [⿴囗十 .

Higher dimensional gauge theories, however, are not asymptotically free. One expects, therefore, that the ultraviolet independence of instanton amplitudes is not a generic feature of compactified higher-dimensional theories, and that nonperturbative effects may receive ultraviolet contributions. That small-instanton amplitudes are modified is clear: as the 
instanton becomes much smaller than the radius of compactification $R$, a large number of Kaluza-Klein (KK) modes begin to "feel" its presence. The KK modes' fluctuations in the small instanton background can grow as the instanton size $\rho$ decreases, modifying the dependence of the instanton amplitude on $\rho$. It is this modification that we study here, extending the work of [5] on nonperturbative effects in compactified supersymmetric theories to theories without supersymmetry.

Our main result is that bulk gauge fields and scalars lead to an exponential, $e^{\mathcal{O}(1) R / \rho}$, enhancement of the small instanton $(\rho \ll R)$ amplitude, while bulk fermions tend to similarly suppress small instantons; which contribution dominates is a detailed question to which we devote the rest of the paper. A qualitative understanding of this result can be obtained by recalling 't Hooft's original calculation [泪] of the instanton amplitude $e^{-S_{\text {eff }}}$ and the relation between instanton amplitudes and beta functions [6, 7]. Nonzero modes of bosons in the instanton background contribute "screening" logarithms of $\rho$ to the effective action $S_{\text {eff. }}$ Screening logarithms decrease ${ }^{1} S_{\text {eff }}$ as $\rho$ is decreased, hence one expects bulk bosons to enhance the small instanton amplitude. On the other hand, nonzero modes of fermions in the instanton background contribute "antiscreening" logarithms that increase the action for small $\rho$, suppressing the small instanton amplitude. The main contribution to the enhancement of the small- $\rho$ amplitude is due to the nonzero modes - the KK fields responsible for the effect are massive and have no zero modes in the instanton background.

To make sense of the calculation of instanton effects in a $5 \mathrm{~d}$ theory, which is neither renormalizable nor asymptotically free, we use deconstruction as an ultraviolet definition of the theory [10, 11]. For the "moose" theory in a $5 \mathrm{~d}$ phase, we use standard $4 \mathrm{~d}$ instanton calculus, renormalization, and regularization of the instanton amplitude. We believe that our conclusion regarding the growth of the small instanton contribution is independent of the ultraviolet completion of the theory. The advantage of deconstruction is that it allows us to exhibit this growth in a controlled, weak coupling approximation (at finite $N$ ), while avoiding formal manipulations with the infinite KK tower.

\subsection{Summary}

In section 2, we begin by recalling some known exact results on nonperturbative effects in compactified 5d supersymmetric theories. In [12, 5] it was shown that the operators violating the anomalous $\mathrm{U}(1)_{R}$ symmetry in the low-energy theory are due to $4 \mathrm{~d}$ instantons wrapped around the compact dimension. The discussion in section 2.1 sets the framework for our calculation, as it is precisely the contribution of wrapped $4 \mathrm{~d}$ instantons in nonsupersymmetric theories that we are interested in.

We continue in section 2.2 by discussing in detail the $\rho$ dependence of the instanton amplitude in a compactified supersymmetric $5 \mathrm{~d}$ theory, using a continuum theory description. We show that, because of supersymmetric cancellations, the dependence of the amplitude on the instanton size is entirely governed by the $4 \mathrm{~d}$ beta function. The instanton amplitude is therefore insensitive to the ultraviolet and its strength is completely determined by $4 \mathrm{~d}$

\footnotetext{
${ }^{1}$ This terminology comes from the relation $S_{\text {eff }}(\rho)=8 \pi^{2} / g^{2}(\rho)$, which holds for the instanton amplitude in massless QCD. A "screening" $\ln \rho$ contribution increases the coupling in the ultraviolet, decreasing $S_{\text {eff }}$ for small $\rho$, and v.v. for the "antiscreening" logarithms. For a review, see [8, 9,
} 
infrared dynamics. This ultraviolet insensitivity of the instanton amplitude in compactified supersymmetric theories explains why the Seiberg-Witten curve of the compactified $5 \mathrm{~d}$ theory, found in [12] without specifying details of the ultraviolet completion, agrees with the deconstructed curve [5].

For a self-contained presentation, in section 3 we give a brief review of deconstruction and the deconstructed semiclassical field configurations whose contributions to the path integral we will consider.

We then turn, in section 1 , to the main goal of this paper: studying the small-instanton dependence of instanton amplitudes in nonsupersymmetric compactified $5 \mathrm{~d}$ gauge theories. We begin with a discussion of our approximation to massive instanton determinants (the "step-function" approximation) in section 4.1. We then use this approximation to calculate the instanton amplitude in the pure gauge theory in section 1.2. We use a deconstructed ultraviolet definition of the theory to regulate and renormalize the amplitude. We show that the amplitude grows exponentially, as $e^{R / \rho}$. Equation (4.10), displaying this behavior of $S_{\text {eff }}$, is the main result of section 4.2 .

We include bulk scalar and fermion fields in the calculation in section 1.3. Within the same approximation as in section 4.2 , we find the dependence of $S_{\text {eff }}$ on the instanton size for arbitrary bulk matter content. Equation (4.14) shows the effect mentioned in the introduction - that bulk bosons enhance, while bulk fermions suppress the small-instanton amplitude.

In section 5, we discuss an important issue: the corrections to our "step-function" approximation to massive instanton determinants and the effect of these corrections on the small-instanton amplitude. Using the small- and large-mass expansions [13, 14 of the instanton determinant, as well as a recently proposed interpolating formula [14], we show that these corrections do not qualitatively alter our conclusions.

\subsection{Outlook}

It is important to stress that our result for the instanton amplitude holds in more general backgrounds - Scherk-Schwarz, orbifold, or warped compactifications, such as (deconstructed) slices of $A d S_{5}$. Thus, they have potential applications to models with Standard Model gauge fields propagating in the bulk of the extra dimensions. We comment on the strength of instanton amplitudes in models with nonsupersymmetric orbifolds of supersymmetric theories at the end of section 4.3 . but leave a detailed investigation of instanton effects in more general backgrounds for future study.

If all Standard Model fermions propagate in the bulk of the extra dimensions, our result indicates that the small instanton amplitude is exponentially suppressed - at least in the finite- $N$, weak-coupling deconstructed case, where our calculation is under control. We can not make a claim as to the strength of instanton-induced processes in the continuum limit. This is a strong-coupling limit of the deconstructed theory, where the semiclassical approximation is not valid. On the other hand, instanton induced interactions are allowed by the symmetries of the theory and one can add the corresponding terms, suppressed by the $5 \mathrm{~d}$ cutoff scale, to the low-energy lagrangian. Our calculation, however, does not allow to determine their coefficient. 
A phenomenologically more interesting situation arises when the Standard Model fermions are localized in extra dimension (so that they do not give rise to KK modes, which suppress the small instanton amplitude). In this case our finite- $N$, weak-coupling calculation indicates that small-instanton processes might be unsuppressed. Furthermore, since the instanton is not localized in the extra dimension, it generates a nonlocal interaction between fermions localized at different positions in the extra dimensions; a more detailed investigation of these effects is under way.

\section{Instantons in compactified 5d gauge theory: the supersymmetric case}

In order to set the framework for our calculation, introduce notation, and discuss its relation to previous work, it is instructive to recall what we know about nonperturbative effects in a compactified 5d supersymmetric Yang-Mills theory.

\subsection{Exact results in compactified 5d supersymmetric theory and semiclassical calculations}

Consider a $5 \mathrm{~d} \mathrm{SU}(2)$ theory with minimal supersymmetry, corresponding to $N=2$ in $4 \mathrm{~d}$. The continuum version of this theory was studied in [12, where the Seiberg-Witten curve was found by symmetry and consistency arguments. The curve determines the low-energy, two-derivative theory of the KK zero modes, including all nonperturbative effects. The continuum and deconstructed version of this theory were studied recently in [0], where a map between the nonperturbative effects in the two descriptions was constructed. It was shown that the nonperturbative contributions to the Seiberg-Witten curve of the compactified $5 \mathrm{~d}$ theory arise, in the semiclassical regime, from $4 \mathrm{~d}$ instantons wrapping the extra dimension.

It is precisely the effect of $4 \mathrm{~d}$ instantons wrapping the compact dimension that we want to study in this paper, in a variety of non-supersymmetric compactified $5 \mathrm{~d}$ theories. The $4 \mathrm{~d}$ instantons give the leading contribution to operators violating classically conserved, but anomalous, quantum numbers: $B-L$ and the Peccei-Quinn symmetry in the Standard Model, or the $\mathrm{U}(1)_{R}$ symmetry of the compactified 5d "Seiberg-Witten" theory [12, 5].

The main issue when performing an instanton calculation is the dependence of the instanton amplitude on the instanton size $\rho$ [ [ Classically, instantons of any size are solutions of the Yang-Mills equations. The instanton size $\rho$ is a collective coordinate, which one integrates over in the path integral. The integral over instanton size $\rho$ classically diverges. However, quantum corrections to the instanton amplitude introduce $\rho$ dependence in the action that helps to determine the typical size of instantons contributing to the amplitude.

The discussion of the previous paragraph holds also in compactified 5d Yang-Mills theory, so long as one considers only the leading two-derivative term in the action: the $4 \mathrm{~d}$ instanton solution independent on the compact coordinate is also a solution of the $5 \mathrm{~d}$ YangMills equations for arbitrary instanton size. The 5d Yang-Mills theory, however, is at best an effective low-energy theory and contains (generally unknown) higher-derivative terms suppressed by inverse powers of the cutoff $M$. These terms can, a priori, determine the 
instanton size already at the classical level (in the manner the Skyrme term determines the size of the skyrmion, or similar higher-dimensional terms determine the size of instantons considered as solitonic particles in a 5d theory, see [15]). However, the higher-derivative terms' contribution to the instanton action is suppressed by inverse powers of $\rho M$, hence for instantons larger than the $5 \mathrm{~d}$ short-distance cutoff, $\rho M \gg 1$, their effect on the action is negligible.

We will see, in section 1 , that the enhancement of the small instanton amplitude in nonsupersymmetric theories occurs for instantons of size $R \gg \rho \gg M^{-1}$, where the higherderivative terms are unimportant; parametrically, at least, the required separation of scales can always be achieved. ${ }^{2}$

In a supersymmetric theory, on the other hand, as we show below (section 2.2), the $\rho$ dependence of the effective action in the instanton background is governed by the $4 \mathrm{~d}$ beta function. In supersymmetric theories, therefore, the instanton amplitude shows no ultraviolet (UV) sensitivity.

\subsection{The $\rho$ dependence of the instanton amplitude in compactified supersym- metric theories}

We are interested in the semiclassical expansion of the $5 \mathrm{~d}$ path integral, formally written in terms of an infinite number of 4d Kaluza-Klein (KK) fields, or the deconstructed version thereof (see section 3), around a $4 \mathrm{~d}$ instanton solution. Semiclassically, the amplitude is proportional to $e^{-S_{\text {class }}}$, where $S_{\text {class }}$ is the classical action of the instanton in the $5 \mathrm{~d}$ theory with coupling $g_{5}$, compactified on a circle of radius $R$ :

$$
S_{\text {class }}=2 \pi R \frac{8 \pi^{2}}{g_{5}^{2}}
$$

Recall now that in the Wilsonean regularization scheme the matching to the coupling, $g_{4}$, of the $4 \mathrm{~d}$ theory, occurs at the UV cutoff $M:^{3}$

$$
\frac{1}{g_{4}^{2}(M)}=\frac{2 \pi R}{g_{5}^{2}}
$$

Therefore, expressed in terms of the $4 \mathrm{~d}$ coupling, the classical action is:

$$
S_{\text {class }}=\frac{8 \pi^{2}}{g_{4}^{2}(M)},
$$

just like in a $4 \mathrm{~d}$ instanton calculation with $g_{4}(M)$ - the "bare" coupling. As usual in instanton calculations, we will take $M$ to be the Pauli-Villars regulator mass. The classical

\footnotetext{
${ }^{2}$ To discuss the effects of instantons of size $\rho \sim M^{-1}$, or even $\rho \ll M^{-1}$, an ultraviolet definition of the theory is needed. In a deconstructed framework, we give a qualitative discussion of the effects of "really small" instantons $\left(\rho \ll M^{-1}\right)$ in section 4.2. In section 4 the cutoff of the $5 \mathrm{~d}$ theory is denoted by $v$, while $M$ is a Pauli-Villars regulator mass; physical quantities in the renormalizable deconstructed theory are independent of $M$.

${ }^{3}$ And not at $1 / R$, as one might naively guess; matching at $1 / R$ is in conflict with holomorphy in $R$ as noted in [16]. This also follows from the RGE (3.7) in the deconstructed version of the theory (with $m_{K K}=2 v \rightarrow M$ ). Note that the matching at the UV cutoff is a general feature of deconstructed regularization of higher dimensional theories even in the absence of supersymmetry.
} 
action is, of course, independent of the instanton size, which is a collective coordinate to be integrated over. However, fluctuations around the instanton depend on its size $\rho$. Studying this dependence is our main goal, since it is crucial in determining the strength of the instanton amplitude.

Let us look first at the fluctuations of the $4 \mathrm{~d}$ gauge field (the KK zero mode) in the instanton background. The nonzero modes of the $n=0 \mathrm{KK}$ states cancel due to supersymmetry [9]. The only contribution is that of the zero modes, changing $e^{-S_{\text {class }}},(2.3)$, to:

$$
\exp \left(-\frac{8 \pi^{2}}{g_{4}^{2}(M)}\right)(\rho M)^{B-F / 2}
$$

where $B, F$ are the number of bosonic and fermionic zero modes respectively. The way (2.4) appears is very simple - the Pauli-Villars regulator determinants have modes with eigenvalue $M^{2}$ for bosons (and $M$ for fermions) whenever there is a zero mode. Since one divides by the PV determinant, regulator boson eigenvalues appear with $+1 / 2$ power and regulator fermions - with $-1 / 2$ (instead of $-1 / 2$ and $1 / 2$, respectively, for physical fields). Combining the above one gets the $B-F / 2$ factor in (2.4).

The important point is that in the supersymmetric case the only $\rho$ dependence appears through the zero modes. There is no $\rho$ anywhere else in the whole Green function (except in the measure, of course). This is to be contrasted with ordinary QCD where the nonzero modes also contribute a $\rho$-dependent factor, as we will see in section 4 . Now, exponentiating the prefactor in (2.4), $S_{\text {class }}$ becomes:

$$
S_{\mathrm{eff}}=\frac{8 \pi^{2}}{g_{4}^{2}(M)}-\left(B-\frac{F}{2}\right) \ln \rho M
$$

The size of the instanton is determined [任 by adding the Higgs action with vev $a$, proportional to $a^{2} \rho^{2}$, and then extremizing w.r.t. $\rho$, which gives $\rho^{2} \sim b_{0} / a^{2}$. Here, $b_{0}$ is the first coefficient of the beta function. In supersymmetric theories $b_{0}=B-F / 2$ precisely because nonzero modes in the instanton background cancel (for the $\mathrm{SU}(2) N=2$ supersymmetric theory we are considering $B=F=8$ ); see [9].

Now we turn to the fluctuations of the KK modes of the gauge field and partners. The spectrum of fluctuations of the heavy modes (with mass $n / R$ ) around the instanton still has an asymmetry between bosons and fermions. This is easiest to see from the fluctuation equations. For example, the KK modes of the gauge field obey the same equation (in the $A_{5}^{n}=0$ background Lorentz gauge) as the $n=0$ mode, up to the mass term:

$$
-D^{2}\left(A^{0}\right) A_{\mu}^{n}+2\left[F_{\mu \nu}^{0}, A^{n \nu}\right]+\frac{n^{2}}{R^{2}} A_{\mu}^{n}=\lambda_{b} A_{\mu}^{n},
$$

where $A^{n}$ is the KK gauge field and $A_{0}, F_{\mu \nu}^{0}$ - the BPST background. If $R$ is infinite, these are the usual $4 \mathrm{~d}$ fluctuation equations in the background Lorentz gauge. Adding the KK mass only shifts the spectrum upwards by $n / R$; the fermions still have eight chiral eigenfunctions with eigenvalues $n / R$ and the bosons - eight bosonic eigenfunctions with eigenvalues $n / R$. There is, therefore, still a net non-cancellation of the lowest eigenvalues 
in the determinants at each KK mass level. Since all eigenvalues larger than $n / R$ come in complete supersymmetry pairs the contributions of all but the lowest eigenvalues cancel in the determinants.

Therefore the fluctuation determinant of the KK gauge fields reduces to the product of the lowest eigenvalues (which would have been the zero modes in the infinite- $R$ limit). Taking these KK gauge fields "zero modes" into account, the instanton amplitude in the supersymmetric $\mathrm{SU}(2)$ theory becomes: ${ }^{4}$

$$
\exp \left(-\frac{8 \pi^{2}}{g_{4}^{2}(M)}+4 \ln (M \rho)\right) \prod_{n=1}^{M R}(n R)^{-8}\left(\sqrt{\frac{n^{2}}{R^{2}}+M^{2}}\right)^{8} .
$$

The first term in the pre-exponential product is the contribution of the would-be zero modes of physical fields and the second term - that of the regulators. ${ }^{5}$ The contribution is to the power of 8 and not 4 because at each KK level there are left and right moving modes, so the number of zero modes of the non-zero KK modes at each level is double that of the $n=0$ zero modes. Equation (2.7) should be multiplied by integrals over the usual fermionic and bosonic zero modes. Since our main interest is the $\rho$ dependence of the measure, we will not explicitly display these integrals here and in the following sections.

It is important to note that the product in (2.7) is independent of $\rho$. We can reexponentiate and obtain:

$$
S_{\text {eff }}=\frac{8 \pi^{2}}{g_{4}^{2}(M)}-4 \ln M \rho-4 \sum_{n=1}^{M R} \ln \left(1+\frac{M^{2} R^{2} \pi^{2}}{n^{2} \pi^{2}}\right)=\frac{8 \pi^{2}}{g_{4}^{2}(1 / R)}-4 \ln \frac{\rho}{R} .
$$

We conclude that the instanton amplitude is proportional to

$$
\exp \left(-S_{\text {eff }}\right)=\exp \left(-\frac{8 \pi^{2}}{g_{4}^{2}(1 / R)}+4 \ln \frac{\rho}{R}\right)=\left(\Lambda_{4} \rho\right)^{4},
$$

where $\Lambda_{4}$ is the $4 \mathrm{~d}$ strong coupling scale of the theory as defined by (2.9).

Equation (2.9) is the main result of this section. It shows that the dependence of the instanton amplitude on the instanton size in the compactified supersymmetric 5d theory is entirely controlled by the $4 \mathrm{~d}$ beta function. The size of the relevant instantons is then determined by the $4 \mathrm{~d}$ infrared (e.g. Higgs) dynamics of the theory. The supersymmetric cancellations of nonzero modes are crucial in establishing this result.

\section{A brief review of deconstruction}

In this section we briefly review the idea of deconstruction - the regularization that we will use to perform the instanton calculation in the compactified nonsupersymmetric theory. Deconstruction has been proposed in [10, 11] as a convenient, explicitly gauge invariant

\footnotetext{
${ }^{4}$ The product in $(2.7)$ should be taken over infinite KK tower. However, we are working in an effective $4 \mathrm{~d}$ theory with a cutoff $M$, and absorbed the contributions of the KK states with mass $m_{n}>M$ into the definition of $g_{4}(M)$. This becomes obvious when the theory is regulated by deconstruction: as long as cutoff (lattice spacing) is finite, the KK tower is finite too.

${ }^{5}$ In the non-supersymmetric case, as we will see in the following sections, we will also advocate that the pre-exponential factors have to be present, based on the singularity of the determinants in the $R \rightarrow \infty$ limit and/or renormalization group invariance with respect to changes of the UV cutoff $M$.
} 
regularization scheme for the study of $D=4+n$ dimensional gauge theories with $n$ compact dimensions. In this paper we study 5 d theories compactified on $S^{1}$ with radius $R$. Their deconstructed description can be obtained by discretizing a single compact dimension. With $N$ lattice sites the discretized lagrangian of a pure gauge theory with an $\mathrm{SU}(M)$ gauge group has the form:

$$
S=\int d^{4} x\left(-\frac{a}{g_{5}^{2}} \sum_{k=1}^{N} \operatorname{tr} F_{k}^{2}+\frac{a}{g_{5}^{2}} \sum_{k=1}^{N} \operatorname{tr}\left(D_{\mu} Q_{k}\right)^{\dagger} D^{\mu} Q_{k}\right),
$$

where $a$ is a lattice spacing, and the link fields $Q$ are proportional to Wilson lines:

$$
Q=\frac{1}{a} e^{i a A_{5}}
$$

It is obvious that the action (3.1) describes a $4 \mathrm{~d}$ product gauge theory with $\mathrm{SU}(M)^{N}$ gauge group and scalar fields $Q$ in the bifundamental representations. More precisely, the product gauge theory description requires that the lagrangian (3.1) be supplemented by a scalar potential $V(Q)$, generating non-vanishing vacuum expectation values (vevs) for the link fields $\langle Q\rangle=v=1 / a$. The dictionary relating the $5 \mathrm{~d}$ and $4 \mathrm{~d}$ parameters is given by:

$$
a=\frac{1}{v}, \quad 2 \pi R=\frac{N}{v}, \quad \frac{1}{g_{5}^{2}}=\frac{v}{g^{2}},
$$

where $g^{2}$ is a gauge coupling of an individual gauge group in product gauge theory. Note that the scalar kinetic term has a non-canonical normalization, $1 / g^{2}$, which is especially convenient in a supersymmetric theory. In fact, one can generalize deconstruction to study supersymmetric theories in 5d [17]. In this case, there is a 4d SYM theory on each lattice site. In the simplest case, when four supercharges are preserved by the latticized theory, the link fields $Q$ are chiral superfields.

Expectation values of the link fields break the product gauge group $\mathrm{SU}(M)^{N}$ to the diagonal $\mathrm{SU}(M)$, giving mass to all but one linear combination of the gauge multiplets. The eigenvalues of the mass matrix are given by:

$$
m_{k}^{2}=4 v^{2} \sin ^{2} \frac{\pi k}{N}, \quad k=0, \ldots N-1 .
$$

One can easily see, using the identifications from eq. (3.3), that, in large- $N$ limit, the KK spectrum of a $5 \mathrm{~d}$ theory is reproduced.

We now turn to the gauge coupling of the unbroken $\mathrm{SU}(M)$ gauge group, which should be identified with the low energy gauge symmetry of the compactified $5 \mathrm{~d}$ theory. The matching of the couplings should be performed at the scale of the highest KK mode $m_{K K}=$ $2 v$ and is given by:

$$
\frac{1}{g_{d}^{2}\left(m_{K K}\right)}=\frac{N}{g^{2}\left(m_{K K}\right)} \text {. }
$$

Note that the continuum (large- $N$ ) limit with fixed $5 \mathrm{~d}$ coupling and radius (which at the same time implies fixed coupling $g_{d}^{2}$ in the low energy theory) requires that:

$$
g^{2}\left(m_{K K}\right) \sim v \sim N \rightarrow \infty .
$$


Equation (3.6) implies that the strong coupling scale of each individual gauge group in the product gauge theory also goes to infinity along with $N$. Hence, in the continuum limit the deconstructed description of the theory is not weakly coupled, reflecting the nonrenormalizability of the original $5 \mathrm{~d}$ model.

On the other hand, the gauge coupling of the diagonal subgroup formally appears asymptotically free and its renormalization group (RG) evolution is given by:

$$
\begin{aligned}
\frac{8 \pi^{2}}{g_{d}^{2}(1 / R)} & =N \frac{8 \pi^{2}}{g^{2}\left(m_{K K}\right)}-b_{0} \ln m_{K K} R+2 b_{0} \sum_{k=1}^{\frac{N-1}{2}} \ln \frac{m_{k}}{m_{K K}} \\
& =N \frac{8 \pi^{2}}{g^{2}\left(m_{K K}\right)}-b_{0} N \ln 2+b_{0} \ln 2 \pi
\end{aligned}
$$

where as before $b_{0}$ is the one-loop beta function coefficient in the low energy theory (e.g., $b_{0}=4$ in the $5 \mathrm{~d} \mathrm{SU}(2) \mathrm{SYM}$ model of section 2 and $b_{0}=7$ in the non-supersymmetric $\mathrm{SU}(2)$ pure gauge theory of section 4.2), and the factor of two in the sum is due to the left and right moving modes. The first line of the RG equation (3.7) is written, for definiteness, for odd $N$ (the second line is true for any $N$ ).

We now turn to the discussion of non-perturbative physics. We are interested in the contributions of instantons in the compactified 5d theory to amplitudes in the low-energy effective theory. In fact, truly localized 5d instantons are not known. However, as has been explained in section 2, topologically non-trivial solutions of the classical equations of motion can be obtained by "lifting" $4 \mathrm{~d}$ instanton solutions to the fifth dimension. Namely, one should consider field configurations which are independent of the fifth coordinate:

$$
A_{\mu}\left(x_{\nu}, x_{5}\right)=A_{\mu}^{\mathrm{inst}}\left(x_{\nu}\right), \quad A_{5}\left(x_{\nu}, x_{5}\right)=0 .
$$

This $5 \mathrm{~d}$ "instanton" is a codimension- 4 object, whose world-line wraps the compact dimension. Clearly, the action is finite so long as the compactification radius $R$ is finite.

We are looking for the description of such instantons in the deconstructed theory. The independence of the compact coordinate in the continuum theory suggests that in the deconstructed description we should look for field configurations independent of position in "theory space" (that is, the gauge fields in all of the gauge groups should have the same profile). In other words, we are looking for a multi-instanton solution with winding numbers $(1,1, \ldots, 1)$ in the individual gauge groups of the $\mathrm{SU}(2)^{N}$ theory. ${ }^{6}$ In the low energy effective theory this field configuration corresponds to a single instanton in the unbroken gauge group, as expected from the wrapped $5 \mathrm{~d}$ instanton picture. Indeed, it was shown in [5] that in the continuum limit this multi-instanton solution corresponds to the $5 \mathrm{~d}$ instanton (3.8). On the other hand, the deconstructed theory has a much richer non-perturbative physics. In particular there exist "fractional" instantons with winding numbers $(1,0, \ldots, 0)$ and so on. Such fractional instantons are lattice artifacts which do not correspond to physical effects in the continuum theory. In [5] it was shown that in supersymmetric theories fractional instanton contributions cancel and do not affect the

\footnotetext{
${ }^{6}$ See $[18]$ for an explanation of this terminology.
} 
low-energy action (ensuring agreement with the continuum theory result [12] for the lowenergy Seiberg-Witten curve). However, one expects that in non-supersymmetric models fractional instantons will affect the low energy properties of the theory. Nevertheless one can use deconstruction to study instanton amplitudes of the 5d theory if one only includes contributions of $(1,1, \ldots, 1)$ instantons to the path integral. It is in this sense that we will treat deconstruction as an UV definition of the $5 \mathrm{~d}$ theory in the following sections.

\section{Instantons in compactified 5d gauge theory: the non-supersymmetric case}

In this section, we study the $\rho$ dependence of the instanton amplitude in a non-supersymmetric compactified $5 \mathrm{~d}$ theory. The main difference from the supersymmetric case is that nonzero modes contribute at all KK levels - without supersymmetry no complete cancellation between fermions and bosons can occur. The results of this section apply to purely non-supersymmetric models as well as to models where the field content is supersymmetric, but the spectrum is not, e.g. Scherk-Schwarz and orbifold models. We assume that the

expectation value of the Wilson line $\mathcal{P} \exp \int_{0}^{2 \pi R} A_{5}(x, y) d y$ vanishes or is small enough to not affect the $\rho \ll R$ behavior of the instanton amplitude (alternatively, the Wilson line can be projected out by orbifolding).

We will consider the instanton amplitude in the pure-gauge (deconstructed) compactified 5d theory in section 4.2 and include bulk matter in section 4.3 . However, we need to first make a detour and briefly discuss the evaluation of KK mode determinants in the instanton background, in particular the "step-function" approximation that we will employ in our calculation. In section 5, we will give a more detailed discussion of the corrections to this approximation and how they affect our results.

\subsection{The "step-function" approximation to massive instanton determinants}

Consider first an instanton in a 4d SU(2) gauge theory with a scalar field of mass $m$, which we take (for definiteness) to be a complex adjoint field. We write down general form of the instanton action as:

$$
S_{\mathrm{eff}}=\frac{8 \pi^{2}}{g^{2}(M)}-\left(8-\frac{2}{3}\right) \ln M \rho+\Delta S_{\mathrm{eff}}(m \rho, M / m)
$$

where $M$ is a UV cutoff (a Pauli-Villars mass, $M \gg m, M \gg \rho$ ), and $\Delta S_{\text {eff }}(m \rho, M / m$ ) is the contribution of the scalar field fluctuations around the instanton. The antiscreening factor of -8 is due to the zero modes of the gauge field in the instanton background, while the screening factor of $+2 / 3$ is from the nonzero modes of the gauge field 四 8 .

To determine $\Delta S_{\text {eff }}(m \rho, M / m)$, we would have to calculate the determinant of scalar fluctuations for arbitrary $m \rho$. This is a technically difficult task - the method of [ : uses the conformal invariance of the massless case and is not applicable to the massive determinant. However, continuity in $\rho$, renormalization group invariance with respect to a change in the cutoff $M$, and the singularities of the determinants expected in the massless limit, will help us find an approximate answer. 
1. For $m \rho \ll 1$, we can neglect the mass and therefore $\Delta S_{\text {eff }}(m \rho, M / m)=\frac{2}{3} \ln M \rho$; the coefficient is the one appropriate for a complex adjoint scalar found in [ $[$. An important point is that $\Delta S_{\text {eff }}(m \rho, M / m)$ has a smooth limit as $m \rightarrow 0$ for fixed $\rho$. This is because the scalar field does not have zero modes in the instanton background even in the massless limit 4 . Such a zero mode would cause a divergence of the instanton amplitude in the $m \rightarrow 0$ limit. This is to be contrasted with the case of a KK gauge field, where bosonic zero modes in the instanton background will appear in the massless limit, causing a singularity in the instanton amplitude; we will see that in the case of massive vector fields $\Delta S_{\text {eff }}$ should be modified to account for the massless limit singularity. There are corrections to the small-mass expression we use: a constant piece, calculated by 't Hooft as well as corrections proportional to positive powers of $m \rho$; these are discussed in section 5, see eq. (5.3) there.

2. For $m \rho \gg 1$, the scalar does not feel the instanton, and therefore $\Delta S_{\text {eff }}(m \rho, M / m)=$ const $(M)$, (independent of $\rho$ ) where the dependence on $M$ is such as to make the action renormalization group invariant with respect to changes of $M$. Corrections suppressed by $1 /(m \rho)$ are certainly present, but are small when $m \rho \gg 1$, see eq. (‥4) of section 5 .

3. Finally, there is another piece of information: quantum mechanically, the action depends on the instanton size $\rho$. It is reasonable to require that the action be a continuous function of $\rho$.

Combining the above arguments, we are led to the following expression for $\Delta S_{\text {eff }}(m \rho, M / m)$ :

$$
\Delta S_{\mathrm{eff}}(m \rho, M / m)=\frac{2}{3} \ln M \rho+\frac{2}{3} g(m \rho)
$$

where $g(m \rho)$ is approximated by:

$$
g(x)= \begin{cases}0, & x<1 \\ -\ln x, & x>1\end{cases}
$$

We will further call eq. (4.3) the "step-function" approximation (despite the obvious continuity of $\Delta S_{\text {eff }}(m \rho, M / m)$ in $\left.\rho\right)$ to the determinant. This is very similar to what one usually does with beta-functions: the states with masses near cutoff do not of course decouple discontinuously as the energy scales are integrated out; yet we discontinuously change the beta function across the threshold and then require that the gauge coupling itself be continuous.

The limits considered above will be important for our study of instanton effects in the (deconstructed) 5d theory. We will show, in section 5, that the "threshold" corrections to the step function approximation (4.3) do not qualitatively modify our conclusions.

\subsection{Deconstructing the instanton amplitude in a compactified pure gauge theory}

With the discussion of the previous section in mind, we are ready to turn to the problem at hand - the strength of instanton effects in the deconstructed non-supersymmetric pure gauge $5 \mathrm{~d}$ theory [10, 11]. We will organize our discussion according to the ratio of the 
instanton size $\rho$ to the "UV cutoff" of the $5 \mathrm{~d}^{\text {theory }}{ }^{7} v$. The scale $v$ is where the $\mathrm{SU}(2)^{N}$ theory is broken down to the diagonal $\mathrm{SU}(2)_{D}$ gauge group (which is to be thought as the " $5 \mathrm{~d}$ " gauge group). We will first consider the contribution of instantons in the far UV region, beyond the cutoff of the " 5 d" theory, i.e. $1 / v \gg \rho$. We will then increase the size of the instanton and will consider the instanton amplitude for intermediate values of $\rho$, $2 \pi R=N / v \gg \rho>1 / v$. This will turn out to be the most interesting, $5 \mathrm{~d}$, regime. Finally, the contribution of large instantons $\rho>R=N /(2 \pi v)$ is governed by the four dimensional beta function and is considered in 沟.

We begin with "really small" instantons, $\rho \ll v^{-1}$, which effectively means $v=0$, i.e. with the moose theory in a "non- $5 \mathrm{~d}$ " phase. In this case, there are $N$ independent instantons of the type $(1,0,0 \ldots),(0,1,0, \ldots)$, etc. Our primary interest, however, are instantons, which appear as solutions of the 5d Yang-Mills equations of motion, independent of the compactified coordinate. In other words, we are interested in instantons of the diagonal gauge group in the moose theory. These are the $(1,1,1,1, \ldots, 1)$-instantons composite objects made out of $N$ instantons, one in each $\mathrm{SU}(2)$ gauge group. The $N$ instanton one-loop effective action for $v=0$ is then (denoting the sizes of various instantons by $\left.\rho_{i}\right)$ :

$$
\prod_{i=1}^{N} \exp \left(-\frac{8 \pi^{2}}{g^{2}(M)}+\left(8-\frac{2}{3}\right) \ln M \rho_{i}-\frac{1}{3} \ln M \rho_{i}\right)=\exp \left(-\frac{8 \pi^{2} N}{g^{2}(M)}+7 \sum_{i=1}^{N} \ln M \rho_{i}\right)
$$

where in the first equation $8-2 / 3$ is the contribution of zero and non-zero modes of the gauge fields, and $-1 / 3$ is the contribution of the bifundamental scalars (they are real) 四. The instantons are independent, so one should integrate over $N$ instanton sizes, centers, and orientations, which makes the $\rho$-integral extremely UV convergent and infrared (IR) divergent. As $v$, breaking $\mathrm{SU}(2)^{N}$ to $\mathrm{SU}(2)_{D}$, is turned on, there is really only one true zero mode; however, for small $v$ one still has $N$ constrained instantons, and the expression (1.4) is a good approximation.

However, when $v \neq 0$, separating instanton centers and/or varying instanton sizes $\rho_{i}$ independently increases the action - the mass term $v^{2} \sum_{i}\left(A_{i}^{\mu}-A_{i-1}^{\mu}\right)^{2}$ increases the action whenever $A_{i}^{\mu} \neq A_{i-1}^{\mu}$. Therefore, one can integrate over $N-1$ instanton sizes and positions (or rather their variations from the "average") and end up with the action of a single $(1,1, \ldots, 1)$ instanton, so that one only integrates over a single $\rho$. From a $5 \mathrm{~d}$ perspective, this is equivalent to considering the contribution of an instanton of the KK zero modes of the $5 \mathrm{~d}$ gauge field.

The integrand for the diagonal instanton of size $\rho \ll 1 / v$ is, in our step-function approximation (4.2):

$$
\exp \left(-\frac{8 \pi^{2} N}{g^{2}(M)}+8 \ln M \rho-\left(1+2 \frac{N-1}{2}\right) \ln M \rho\right) \times \prod_{k=1}^{\frac{N-1}{2}}\left(\frac{\sqrt{m_{k}^{2}+M^{2}}}{m_{k}}\right)^{16} .
$$

\footnotetext{
${ }^{7}$ The 5d "UV cutoff" $v$ is not to be confused with the true cutoff of the $4 \mathrm{~d}$ deconstructed description, the Pauli-Villars mass $M$, which we take to be larger than $v$.
} 
The various factors in (4.5) arise as follows:

1. The antiscreening logarithm $8 \log M \rho$ reflects the contribution of the true zero modes of the diagonal unbroken gauge group.

2. The screening logarithm comes, as usual, from the contribution of non-zero modes of both massless and KK states - recall that $\rho \ll 1 / v$, therefore all states are lighter than $1 / \rho$ and feel the presence of the instanton. More precisely, the screening contribution from the massless states - the diagonal gauge bosons' nonzero modes as well as the massless real adjoint scalars' nonzero modes - gives a total factor of $-1=-2 / 3-1 / 3$. The $\frac{N-1}{2}$ (for simplicity $N$ is taken odd here) doubly degenerate (left and right movers) KK levels contribute each the same factor of $-1(=-2 / 3-1 / 3)$, resulting in the second term in the screening logarithm.

3. The pre-exponential factor in $(4.5)$ is due to the gauge field KK states. It reflects the fact that the $m_{k} \rightarrow 0$ limit of the KK gauge field determinant is singular because of the 16 zero eigenvalues appearing at each KK level; recall that the KK mass levels are doubly degenerate $(16=2 \times 8)$. The additive factor of $m_{k}^{2}$ in the numerator should, of course, be dropped since the UV cutoff $M$ is above any of the KK masses. The pre-exponential term in (4.5) was written in a manner underscoring the analogy with the supersymmetric case, where the lowest eigenvalues are the only ones contributing. Similarly, here the pre-exponential term can also be thought of as the contribution of the lowest eigenvalue - the one vanishing in the massless limit — of the KK gauge field determinant, ensuring the correct singular behavior in the $m_{k} \rightarrow 0$ limit. Further corrections to the amplitude (4.5) should vanish in the massless limit and are proportional to positive powers of $m_{k} \rho$ (see section 5).

4. Finally, it is easily verified that (4.5) is RG invariant with respect to change of UV cutoff $M$; the relevant RG equation is the one for the individual $\mathrm{SU}(2)_{i}$ gauge groups, since $M$ is above any mass thresholds.

Let us now increase size of the instanton. As $1 / \rho$ crosses any given threshold $m_{k}$, the corresponding states decouple from the instanton, and the contribution of their non-zero modes simply turns from $-2 \ln M \rho$ into $-2 \ln \left(M / m_{k}\right)$, as in eq. (4.2) in the step function approximation (4.3). Therefore, denoting by $K(\rho)$ the number of KK levels lighter than $1 / \rho$, the instanton amplitude (4.5) turns into:

$$
\exp \left(-\frac{8 \pi^{2} N}{g^{2}(M)}+8 \ln M \rho-(1+2 K(\rho)) \ln M \rho\right) \times \prod_{k=1}^{K(\rho)}\left(\frac{M}{m_{k}}\right)^{16} \prod_{k=K(\rho)+1}^{\frac{N-1}{2}}\left(\frac{M}{m_{k}}\right)^{14}
$$

The pre-exponential factor for the gauge fields heavier than $1 / \rho$ is required by $\mathrm{RG}$ invariance of the amplitude with respect to changes of $M$; since states with mass greater than $1 / \rho$ do not feel the instanton, the prefactor does not depend on $\rho$ (up to the already mentioned corrections in the action, suppressed by inverse powers of $\rho m_{k>K(\rho)}$ or positive powers of $\left.\rho m_{k<K(\rho)}\right)$. 
We then exponentiate the prefactor in (4.6), and after some simple algebraic manipulations find the instanton amplitude $e^{-S_{\text {eff }}}$, with $S_{\text {eff }}$ given by: ${ }^{8}$

$$
S_{\text {eff }}=\frac{8 \pi^{2}}{g^{2}(1 / R)}-7 \ln \frac{\rho}{R}+2 \sum_{k=1}^{K(\rho)} \ln m_{k} \rho .
$$

To arrive at (4.7), we used RG invariance with respect to the cutoff $M$ to replace $M$ with $m_{K K}=2 v$ in (4.6) and then applied the deconstructed theory RG equation (i.e., eq. (3.7) written for the theory under consideration):

$$
\frac{8 \pi^{2}}{g^{2}\left(m_{K K}\right)}=\frac{8 \pi^{2}}{g^{2}(1 / R)}+7 \ln m_{K K} R-14 \sum_{j=1}^{\frac{N-1}{2}} \ln \frac{m_{j}}{m_{K K}}=\frac{8 \pi^{2}}{g^{2}(1 / R)}+7 N \ln 2-7 \ln 2 \pi,
$$

to express $g\left(m_{K K}\right)$ in terms of $g\left(\frac{1}{R}=\frac{2 \pi v}{N}\right)$. The latter, low-energy coupling is the one to be kept fixed in physical applications.

Now let us consider $S_{\text {eff }}$ of eq. (4.7) in two cases: either by taking the usual, nondeconstructed, expressions for KK masses or by using the deconstructed formula. When $K(\rho)$ is (moderately, see below) large we can also approximate $K(\rho)=[R / \rho]$.

We begin by taking $m_{k}=k / R$ - a case which is simple to analyze analytically. The action (4.7) becomes:

$$
S_{\mathrm{eff}}=\frac{8 \pi^{2}}{g^{2}(1 / R)}-7 \ln \frac{\rho}{R}+2 K(\rho) \ln \frac{\rho}{R}+2 \ln K(\rho) !
$$

Substituting $K(\rho)=[R / \rho]$ and using Stirling's formula to perform the sum, we obtain for the action:

$$
\begin{aligned}
S_{\mathrm{eff}}(R \gg \rho \gg 1 / v) & =\frac{8 \pi^{2}}{g^{2}(1 / R)}-6 \ln \frac{\rho}{R}+2 \frac{R}{\rho} \ln \frac{\rho}{R}+2 \frac{R}{\rho} \ln \frac{R}{\rho}-2 \frac{R}{\rho} \\
& =\frac{8 \pi^{2}}{g^{2}(1 / R)}-2 \frac{R}{\rho}-6 \ln \frac{\rho}{R} .
\end{aligned}
$$

It is obvious that the log-enhanced terms cancel (except for low-energy contributions coming from physics below compactification scale). Most importantly, we note that the term linear in $1 / \rho$ decreases the action for small $\rho$, enhancing the small instanton contribution.

We should stress that eq. (4.10) is a valid approximation to the deconstructed expression provided: i.) $1 / \rho$ is sufficiently below the top of the KK tower so that the expressions for the KK masses are approximately correct and ii.) $R / \rho \geq O(10)$, so that there is a large number of KK modes lighter than $1 / \rho$ that feel the instanton. The conclusion we can draw from (4.10), is that for instanton size much smaller than $R$ the effective action decreases as $\rho$ becomes smaller. Thus, the instanton amplitude grows in the UV. Equation (4.10) demonstrates the UV sensitivity of instanton induced amplitudes in extra dimensional gauge theories.

\footnotetext{
${ }^{8}$ From now on, unless otherwise noted, $g$ stands for the coupling of the diagonal group.
} 
Earlier in this section, in our qualitative discussion of deconstructed instanton amplitudes, we argued that for $\rho \ll 1 / v$ the action again becomes an increasing function of $1 / \rho$ (more precisely, of the $N$ independent sizes $\rho_{i}$ of the $N$ instantons that the diagonal instanton "dissociates" into for $\rho \ll 1 / v)$. Together with our analysis of the KK case of the previous paragraph, this allows us to conclude that there is an "extra-dimensional" saddle point of the $\rho$ integral, presumably of order the inverse cutoff scale.

In order to study the effects of instantons with sizes near the inverse cutoff of the deconstructed theory in more detail, we will consider the more appropriate deconstructed expressions for the KK masses: $m_{k}=2 v \sin \frac{\pi k}{N}, k=0, \ldots, \frac{N-1}{2}$. To study the $\rho$ dependence for smaller values of $\rho$ we proceed to evaluate eq. (4.7) numerically. We find that the $\rho$ dependent part of $S_{\text {eff }}$ becomes negative at about $R / \rho \sim 10$, for all values ${ }^{9}$ of $N$, consistent with (and implied by!) the KK analysis above. Further decreasing the value of $\rho$, the action decreases to further negative values (see figure 1), somewhat faster than the KK approximation 4.10 would suggest.

To summarize, our calculation indicates that instanton amplitudes in extra dimensional gauge theories grow with decreasing instanton size. The strength of the instanton-induced interaction is thus decided by the UV completion of the theory. Using deconstruction as the UV completion of the $5 \mathrm{~d}$ theory requires a strong coupling analysis in the large- $N$ continuum limit. Since the instanton vertex is allowed by the symmetries of the theory, one would expect it to appear in the low energy theory suppressed by inverse powers of $v$,

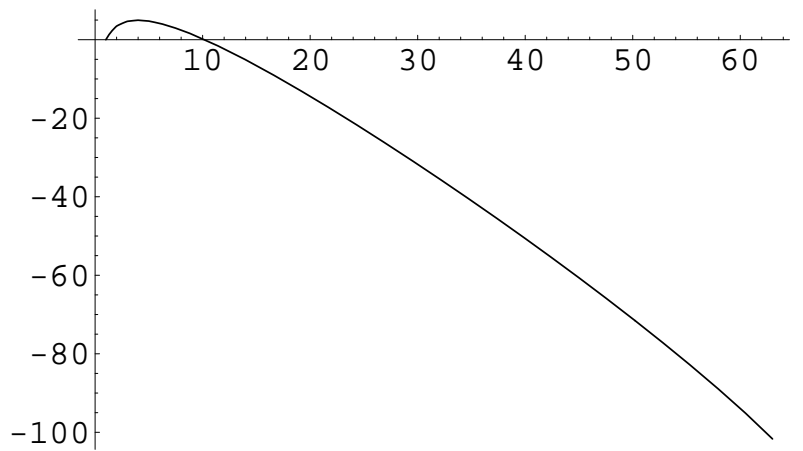

Figure 1: The $\rho$-dependent part of $S_{\text {eff }}$, 4.7), in the deconstructed $(N=200) 5 \mathrm{~d} \mathrm{SU}(2)$ pure gauge theory: $S_{\text {eff }}-8 \pi^{2} / g^{2}(1 / R)$ for $1<R / \rho<N / \pi$. the cutoff of the $5 \mathrm{~d}$ theory. For a field theory in the strong coupling regime we do not, generally, expect higher order corrections (even though they are non-calculable) to conspire to make some operators smaller than their "natural" (as determined by dimensional analysis) value.

Similarly, in more general UV completions of the 5d theory (such as 5d compactifications of string theory) one expects that short distance degrees of freedom may contribute to amplitudes involving extremely small instantons $\left(\rho<g_{5}^{2}\right)$. However, generically short distance physics will not suppress ${ }^{10}$ the contribution of the low-energy degrees of freedom to the instantons of the size $\rho>g_{5}^{2}$.

Finally, we note that the results of this section are not qualitatively changed by orbifolding the $5 \mathrm{~d}$ pure gauge theory (e.g. projecting out the zero mode of $A_{5}$ and taking only

\footnotetext{
${ }^{9}$ Note that large values of $N$ are required in order to observe this effect: the validity of (4.10) already requires $\rho \gg 1 / 2 v$, i.e. $N / \pi \gg R / \rho>O(10)$; for the theory at hand, numerically the effect appears at $N \sim 60$.

${ }^{10}$ Theories with UV/IR correspondence may give a counter example; yet such vacua are non-generic.
} 
half the KK spectrum), even though the precise coefficients in $S_{\text {eff }}$ do (trivially) change. In the next section, we consider the effect matter fields have on the instanton amplitude.

\subsection{Bulk matter and the instanton amplitude}

It is easy to generalize the above calculation to an arbitrary matter content in the bulk of the extra dimension. More precisely, we will consider a theory with $N_{F}^{a}$ adjoint $5 \mathrm{~d}$ fermions (i.e. two $4 \mathrm{~d}$ adjoint Weyl fermion KK zero modes), $N_{F}^{f}$ fundamental 5 d fermions $\left(2 N_{F}\right.$ fundamental Weyl 4d fermion KK zero modes), $N_{S}^{a}$ real adjoint scalars, and $N_{S}^{f}$ complex fundamental scalars.

Equation (4.6) for the instanton amplitude for $R>\rho>1 / 2 v$ is trivially generalized to include the above matter content:

$$
\begin{aligned}
& \exp \left[-\frac{8 \pi^{2}}{g^{2}(M)}+\left(7-\frac{8}{3} N_{F}^{a}-\frac{1}{3} N_{S}^{a}-\frac{2}{3} N_{F}^{f}-\frac{1}{6} N_{S}^{f}\right) \ln M \rho+\right. \\
& \left.+\left(-2+\frac{8}{3} N_{F}^{a}-\frac{2}{3} N_{S}^{a}+\frac{2}{3} N_{F}^{f}-\frac{1}{3} N_{S}^{f}\right) K(\rho) \ln M \rho\right] \times \\
& \times \prod_{k=1}^{K(\rho)}\left(\frac{M}{m_{k}}\right)^{16-8 N_{F}^{a}-2 N_{F}^{f}} \times \prod_{k=K(\rho)+1}^{\max }\left(\frac{M}{m_{k}}\right)^{14-\frac{16}{3} N_{F}^{a}-\frac{4}{3} N_{F}^{f}-\frac{2}{3} N_{S}^{a}-\frac{1}{3} N_{S}^{f}} .
\end{aligned}
$$

For simplicity, we assumed that all fields have the same KK spectra given by $m_{k}, k \geq 1$. Generalizing (4.11) for nonequal mass spectra, occurring, e.g., in Scherk-Schwarz/orbifold type symmetry breaking is easy: one simply has to separate the preexponential terms corresponding to fields with different spectra and, after exponentiating, perform the sums separately.

The amplitude (4.11) was derived as in the pure gauge case. The first product in the preexponential reflects the additional fact that in the massless limit the fermion determinants vanish, while the second product is as required by RG invariance. The coefficients for the contributions of the nonzero modes to the instanton amplitude for massless scalars and fermions in the adjoint and fundamental representations are taken from [ [ we are using the step-function approximation (1.2) for the massive determinants. For completeness, we give the contributions of the zero and nonzero modes of the massless fields of interest in the table 1. From table 1 the amplitude (4.11) is easily recovered using the considerations of the previous section.

\begin{tabular}{|l|c|c|}
\hline field, representation & zero modes & nonzero modes \\
\hline gauge, adjoint & 8 & $-2 / 3$ \\
scalar, real adjoint & 0 & $-1 / 3$ \\
scalar, fundamental & 0 & $-1 / 6$ \\
fermion, Weyl adjoint & -2 & $2 / 3$ \\
fermion, Weyl fundamental & $-1 / 2$ & $1 / 6$ \\
\hline
\end{tabular}

Table 1: The coefficient $c$, in terms of which the contribution of the determinant of the corresponding field to the instanton amplitude is $\exp [c \ln M \rho]$ 
Writing the amplitude as $e^{-S_{\text {eff }}}$ and exponentiating the prefactors, we obtain for the effective action:

$$
\begin{aligned}
S_{\text {eff }}= & \frac{8 \pi^{2}}{g^{2}(M)}+\left(-7+\frac{8}{3} N_{F}^{a}+\frac{1}{3} N_{S}^{a}+\frac{2}{3} N_{F}^{f}+\frac{1}{6} N_{S}^{f}\right) \ln M R+ \\
& +\left(-14+\frac{16}{3} N_{F}^{a}+\frac{2}{3} N_{S}^{a}+\frac{4}{3} N_{F}^{f}+\frac{1}{3} N_{S}^{f}\right) \sum_{k=1}^{\max } \ln \frac{M}{m_{k}}+ \\
& +\left(-7+\frac{8}{3} N_{F}^{a}+\frac{2}{3} N_{F}^{f}+\frac{1}{3} N_{S}^{a}+\frac{1}{6} N_{S}^{f}\right) \ln \frac{\rho}{R}+ \\
& +\left(2-\frac{8}{3} N_{F}^{a}-\frac{2}{3} N_{F}^{f}+\frac{2}{3} N_{S}^{a}+\frac{1}{3} N_{S}^{f}\right) \sum_{k=1}^{K(\rho)} \ln m_{k} \rho .
\end{aligned}
$$

Let us now make some observations on the form of $S_{\text {eff. }}$. We first note that eq. (4.12) can be used to reproduce the $\rho$-dependence of the instanton amplitude in the supersymmetric case; agreement with supersymmetry provides a useful check on our formulae. A supersymmetric $5 \mathrm{~d} \mathrm{SU}(2)$ theory with $n_{a}$ adjoint and $n_{f}$ fundamental matter hypermultiplets corresponds to taking $N_{F}^{a}=1+n_{a}, N_{S}^{a}=1+4 n_{a}$, and $N_{S}^{f}=2 N_{F}^{f}=2 n_{f}$. In the supersymmetric case, $S_{\text {eff }}$ drastically simplifies - all $\rho$ dependence is governed by the $4 \mathrm{~d}$ beta function coefficient, equal to $-4+4 n_{a}+n_{f}$, as in the pure $5 \mathrm{~d}$ SYM case we started with:

$$
S_{\mathrm{eff}}^{S U S Y}=\frac{8 \pi^{2}}{g^{2}(M)}+\left(-4+4 n_{a}+n_{f}\right)\left[\ln M R+\sum_{k=1}^{\max } \ln \frac{M^{2}}{m_{k}^{2}}+\ln \frac{\rho}{R}\right],
$$

the contribution of the nonzero modes having cancelled out as in (2.9). The role of the $\rho$-independent terms in (4.13) is to change the argument of the coupling $M \rightarrow 1 / R$, as in (2.9), 4.7).

We can write eq. (4.12) in terms of the low-energy $4 \mathrm{~d}$ coupling $g(1 / R)$ as:

$$
\begin{aligned}
S_{\mathrm{eff}}= & \frac{8 \pi^{2}}{g^{2}(1 / R)}+\left(-7+\frac{8}{3} N_{F}^{a}+\frac{2}{3} N_{F}^{f}+\frac{1}{3} N_{S}^{a}+\frac{1}{6} N_{S}^{f}\right) \ln \frac{\rho}{R}+ \\
& +\left(2-\frac{8}{3} N_{F}^{a}-\frac{2}{3} N_{F}^{f}+\frac{2}{3} N_{S}^{a}+\frac{1}{3} N_{S}^{f}\right) \sum_{k=1}^{K(\rho)} \ln m_{k} \rho .
\end{aligned}
$$

Equation (4.14) is the main result of this section. It allows us to study the $\rho$ dependence of the instanton amplitude. As in the pure gauge case, the large- $R / \rho$ behavior is controlled by the last term, since it is the one leading to a linear dependence of the action on $R / \rho$. We can see the behavior at small $\rho$ already in the KK approximation: if the coefficient of the last term is positive, the effective action is an decreasing function for sufficiently large $R / \rho$, as in the pure gauge case.

The general effect of bulk matter fields is clear from (4.14): bulk fermions tend to suppress the contributions of small instantons, while bulk bosons tend to enhance them. Precisely how the balance in (dis-)favor of small instantons is achieved depends on the matter content of the theory. For example, for a $5 \mathrm{~d} \mathrm{SU}(2) \mathrm{YM}$ theory with $N_{F}$ fundamental 
fermions, equivalent to $N_{F}$ fundamental flavors in $4 \mathrm{~d}$, we find from eq. (4.12) that if $N_{F}<3$, the instanton amplitude grows for small $\rho$, while for $N_{F} \geq 3$, the amplitude is dominated by the $4 \mathrm{~d}$ saddle point.

As mentioned in the beginning of this section, eq. (4.14) is also applicable for spectra different from the usual KK spectra, e.g. for orbifold compactifications where different fields have different KK expansions. For a $4 \mathrm{~d}$ theory obtained as an orbifold of a $5 \mathrm{~d}$ theory, one can draw similar conclusions - that bulk bosons enhance the small instanton contribution, while bulk fermions suppress it.

If one is considering an orbifold of a supersymmetric theory, one expects additional significant cancellations in (4.12). To see this, note that in the supersymmetric limit the term responsible for the growth of the amplitude for small $\rho$ (the last term in $S_{\text {eff }}$ ) vanishes. If the supersymmetric theory is orbifolded, one expects the cancellations between bosons and fermions to still persist, leaving at most a logarithmic dependence on $\rho$. To this end, consider the contribution to the instanton amplitude of a fundamental matter hypermultiplet, where, in the KK approximation, the scalars have mass $m_{k}^{s}=(k+\theta) / R$ and the fermions $-m_{k}^{f}=k / R$, so that only the fermions have KK zero modes. Clearly, then, the contribution of the hypermultiplet to the last term in (4.12) becomes, dropping possible $\sim \ln \rho$ contributions arising from the "edges" of the spectrum:

$$
\frac{2}{3} \sum_{k=1}^{[R / \rho]} \ln \frac{k+\theta}{k} \simeq \frac{2}{3} \theta \ln \frac{R}{\rho} .
$$

Similar cancellations will occur in the other orbifolded supermultiplets, resulting at most in a logarithmic growth of the amplitude as a function of instanton size.

Using (4.14), one can also study instanton effects in even more general backgrounds where gauge fields propagate, such as a slice of (deconstructed) $A d S_{5}$. A more detailed investigation of the implications of (4.14) in different models will be given elsewhere.

\section{Corrections to the step-function approximation}

In this section, we will consider the role of the corrections to the step-function approximation (4.3) we used throughout the previous sections. We will show that they do not qualitatively alter our conclusions.

Consider first a bulk scalar field in the fundamental representation, whose nonzero KK modes give the following contribution ${ }^{11}$ to $S_{\text {eff }}$ (as usual, the amplitude is $\sim e^{-S_{\text {eff }}}$ ):

$$
\Delta S_{\mathrm{eff}}=\frac{1}{3} \sum_{k=1}^{\max }\left(\ln M \rho+g\left(m_{k} \rho\right)\right) .
$$

In the step function approximation of eq. (4.3) this gives rise to the contribution shown in (4.12).

\footnotetext{
${ }^{11}$ Note that we are taking twice the contribution from the table, because at each mass level there are two scalars.
} 
Let us now subtract the $\rho$-independent terms (representing the contribution of the scalar to the change of the scale $M \rightarrow 1 / R$ of the gauge coupling) from the scalar contribution to the effective action $\Delta S_{\text {eff }}$ (5.1):

$$
\Delta S_{\mathrm{eff}}^{\prime} \equiv \Delta S_{\mathrm{eff}}-\frac{1}{3} \sum_{k=1}^{\max } \ln \frac{M}{m_{k}}=\frac{1}{3} \sum_{k=1}^{\max }\left(g\left(m_{k} \rho\right)+\ln m_{k} \rho\right) .
$$

$\Delta S_{\text {eff }}^{\prime}$ from eq. (5.2) gives the $\rho$-dependent part of the contribution of the KK tower to the instanton action with the gauge coupling evaluated at $1 / R$. If we substitute (1.3), $g(x)=-\theta(x-1) \ln x$, into (5.2), we arrive at the effective action in the step-function approximation: only the light fields contribute to $\Delta S_{\text {eff }}^{\prime}$, giving a large negative $\left(m_{k} \rho<1\right)$ contribution to $S_{\text {eff }}$ in (4.14).

Here, we are interested in the corrections to the step-function approximation (4.3) to $g(m \rho)$ and the effect of the corrections on the strength of the amplitude. The small-mass expansion, ${ }^{12}$ valid for $x \leq .5$, of the determinant is, as shown in [13, 14]:

$$
\frac{1}{6} g(x)=c-\frac{1}{2} x^{2}\left(\ln \frac{1}{x}+d\right)+\mathcal{O}\left(x^{4}\right)
$$

where $c=0.145873, d=0.005797$. The large-mass expansion, $x \geq 1.8$, computed to order $x^{-8}$ in 14], is:

$$
\frac{1}{6} g(x)=-\frac{1}{6} \ln x-\frac{1}{75 x^{2}}-\frac{17}{735 x^{4}}+\cdots
$$

Note that, quantitatively, the constant $c$ in the small-mass expansion is the largest error in the approximation (4.3): even if we define the step-function approximation so that $g(x)=6 c$ for all $0<x<1$, the conclusion that the scalar field fluctuations enhance the amplitude is not altered (since $c<1 / 6$ ). It should be noted, however, that such a modification of $g(x)$ grossly overestimates the error of the step-function approximation, since, as the small $x$ expansion (5.3) shows, $g(x)$ is a decreasing function of $x$.

We can be more precise in our estimate of the error if we use the interpolating function for $g(x)$, proposed in [14] under the plausible assumption that the determinant is a smooth function of $x$, monotonically connecting the large- and small-mass limits:

$$
\frac{1}{6} g(x)_{\text {interp. }}=\frac{0.145873-0.443416 x^{2}-\frac{x^{4}}{5}+\frac{\ln x}{6}}{1-3 x^{2}+20 x^{4}+15 x^{6}}-\frac{\ln x}{6},
$$

which has the correct expansions (5.3), (5.4) for both large and small $x$. We then use (5.5) to numerically perform the sum ${ }^{13}$ over the deconstructed mass spectrum in (5.2) and find the following $\rho$-dependence of $\Delta S_{\text {eff }}^{\prime}$ :

$$
\left.\Delta S_{\text {eff }}^{\prime}\right|_{\text {interp. }} \approx-0.22 \frac{R}{\rho},
$$

\footnotetext{
${ }^{12}$ We multiplied $g$ by the factor $1 / 6$ in $(5.3), 5.4$ in order to simplify comparison with 13, 14, where the determinant of a single fundamental scalar, contributing $g / 6$, was considered.

${ }^{13}$ As in section 1 , for the $\mathrm{SU}(2)$ theory at hand, one needs $N>60$; the linear dependence appears already for $R / \rho>\mathcal{O}(10)$.
} 
where the coefficient has a slight dependence of $R / \rho$ (becoming weaker as $R / \rho$ is increased). We can now compare this contribution to the result found in the step-function approximation with KK spectrum, i.e. eq. (4.10) adapted to the scalar case by replacing $2 \rightarrow 1 / 3$ :

$$
\left.\Delta S_{\text {eff }}^{\prime}\right|_{\text {step }, K K}=-\frac{1}{3} \frac{R}{\rho} .
$$

We conclude that $\Delta S_{\text {eff }}^{\prime}$ is a linearly decreasing function of $R / \rho$ even when threshold corrections are taken into account (albeit with a somewhat reduced coefficient ${ }^{14}$ when compared to step-function approximation).

The fact that corrections do not modify our conclusion qualitatively holds also for scalars in other representations as well as for fermions and gauge fields. Indeed, the propagators for massive fermions and gauge fields can be related to those of the scalar fields [19. In particular, the contribution of any massive state to the effective action is the same as that of a scalar up to overall spin- and isospin-dependent factor (and to the possible wouldbe zero mode contributions) [14]. Thus, properly including the contributions of the massive thresholds, we obtain an improved expression for the one-loop effective action (4.14):

$$
\begin{aligned}
S_{\text {eff }}= & \frac{8 \pi^{2}}{g^{2}(1 / R)}+\left(-7+\frac{8}{3} N_{F}^{a}+\frac{2}{3} N_{F}^{f}+\frac{1}{3} N_{S}^{a}+\frac{1}{6} N_{S}^{f}\right) \ln \frac{\rho}{R}+ \\
& +\left(2-\frac{8}{3} N_{F}^{a}-\frac{2}{3} N_{F}^{f}+\frac{2}{3} N_{S}^{a}+\frac{1}{3} N_{S}^{f}\right) \sum_{k=1}^{\max }\left(g\left(m_{k} \rho\right)+\ln m_{k} \rho\right),
\end{aligned}
$$

with $g(m \rho)$ given by the interpolating function (5.5).

\section{Acknowledgments}

It is a pleasure to thank Csaba Csáki, Josh Erlich, and Yael Shadmi for collaboration at the early stages of this project and for many useful discussions. E.P. thanks Nima ArkaniHamed, Andy Cohen, and Sergei Khlebnikov for discussions and insightful remarks. Y.S. is also grateful to Markus Luty, Sandip Pakvasa, and Xerxes Tata for useful discussions. Y.S. is supported in part by DOE grant DE-FG03-92-ER-40701.

\section{References}

[1] N. Arkani-Hamed, S. Dimopoulos and G.R. Dvali, The hierarchy problem and new dimensions at a millimeter, Phys. Lett. B 429 (1998) 263 hep-ph/9803315;

I. Antoniadis, N. Arkani-Hamed, S. Dimopoulos and G.R. Dvali, New dimensions at a millimeter to a Fermi and superstrings at a TeV, Phys. Lett. B 436 (1998) 257 hep-ph/9804398;

L. Randall and R. Sundrum, A large mass hierarchy from a small extra dimension, Phys. Rev. Lett. 83 (1999) 3370 hep-ph/9905221.

\footnotetext{
${ }^{14}$ The change in the coefficient is mostly due to the better approximation (5.5); the result is insensitive to whether deconstructed or KK spectrum is used, so long as $1 / \rho$ is sufficiently below the top of the KK tower.
} 
[2] L. Randall and R. Sundrum, Out of this world supersymmetry breaking, Nucl. Phys. B 557 (1999) 79 hep-th/9810155;

D.E. Kaplan, G.D. Kribs and M. Schmaltz, Supersymmetry breaking through transparent extra dimensions, Phys. Rev. D 62 (2000) 035010 hep-ph/9911293;

Z. Chacko, M.A. Luty, A.E. Nelson and E. Ponton, Gaugino mediated supersymmetry breaking, J. High Energy Phys. 01 (2000) 003 hep-ph/9911323;

R. Barbieri, L.J. Hall and Y. Nomura, A constrained standard model from a compact extra dimension, Phys. Rev. D 63 (2001) 105007 hep-ph/0011311.

[3] N. Arkani-Hamed and S. Dimopoulos, New origin for approximate symmetries from distant breaking in extra dimensions, Phys. Rev. D 65 (2002) 052003 hep-ph/9811353;

N. Arkani-Hamed and M. Schmaltz, Hierarchies without symmetries from extra dimensions, Phys. Rev. D 61 (2000) 033005 hep-ph/9903417.

[4] G. 't Hooft, Computation of the quantum effects due to a four-dimensional pseudoparticle, Phys. Rev. D 14 (1976) 3432; erratum ibid. 18 (1976) 2199.

[5] C. Csáki et al., Exact results in 5D from instantons and deconstruction, Phys. Rev. D 65 (2002) 085033 hep-th/0110188.

[6] V.A. Novikov, M.A. Shifman, A.I. Vainshtein and V.I. Zakharov, Exact Gell-Mann-Low function of supersymmetric Yang-Mills theories from instanton calculus, Nucl. Phys. B 229 (1983) 381 .

[7] V.A. Novikov, M.A. Shifman, A.I. Vainshtein and V.I. Zakharov, Beta function in supersymmetric gauge theories: instantons versus traditional approach, Phys. Lett. B 166 (1986) 329 Sov. J. Nucl. Phys. 43 (1986) 294.

[8] A.I. Vainshtein, V.I. Zakharov, V.A. Novikov and M.A. Shifman, ABC of instantons, Sov. Phys. Usp. 24 (1982) 195 Usp. Fiz. Nauk. 136 (1982) 553.

[9] M.A. Shifman and A.I. Vainshtein, Instantons versus supersymmetry: fifteen years later, hep-th/9902018.

[10] N. Arkani-Hamed, A.G. Cohen and H. Georgi, (De)constructing dimensions, Phys. Rev. Lett. 86 (2001) 4757 hep-th/0104005.

[11] C.T. Hill, S. PoKörski and J. Wang, Gauge invariant effective lagrangian for Kaluza-Klein modes, Phys. Rev. D 64 (2001) 105005 hep-th/0104035;

H.-C. Cheng, C.T. Hill, S. PoKörski and J. Wang, The standard model in the latticized bulk, Phys. Rev. D 64 (2001) 065007 hep-th/0104179.

[12] N. Nekrasov, Five dimensional gauge theories and relativistic integrable systems, Nucl. Phys. B 531 (1998) 323 hep-th/9609219.

[13] R.D. Carlitz and D.B. Creamer, Light quarks and instantons, Ann. Phys. (NY) 118 (1979) 429 .

[14] O.-K. Kwon, C.-K. Lee and H. Min, Massive field contributions to the QCD vacuum tunneling amplitude, Phys. Rev. D 62 (2000) 114022 hep-ph/0008028.

[15] C.T. Hill, Topological solitons from deconstructed extra dimensions, Phys. Rev. Lett. 88 (2002) 041601 hep-th/0109068.

[16] M.A. Luty and R. Sundrum, Radius stabilization and anomaly-mediated supersymmetry breaking, Phys. Rev. D 62 (2000) 035008 hep-th/9910202. 
[17] C. Csáki, J. Erlich, C. Grojean and G.D. Kribs, 4d constructions of supersymmetric extra dimensions and gaugino mediation, Phys. Rev. D 65 (2002) 015003 hep-ph/0106044.

[18] C. Csáki and H. Murayama, Instantons in partially broken gauge groups, Nucl. Phys. B 532 (1998) 498 hep-th/9804061.

[19] L.S. Brown and C.-K. Lee, Massive propagators in instanton fields, Phys. Rev. D 18 (1978) 2180 . 